Copyright (C) 2014 by Academic Publishing House Researcher



Published in the Russian Federation

European Researcher

Has been issued since 2010.

ISSN 2219-8229

E-ISSN 2224-0136

Vol. 81, No. 8-2, pp. 1488-1490, 2014

DOI: 10.13187/er.2014.81.1488

www.erjournal.ru



Physical and Mathematical sciences

Физико-Математические науки

\title{
The Fourth Main Boundary Value Problem of Dynamics of Thermo-resiliency's Momentum Theory
}

\author{
${ }^{1}$ Merab Aghniashvili \\ 2 Diana Mtchedlishvili
}

1-2 Iakob Gogebashvili Telavi State University, Georgia

2200 Telavi, 1, Kartuli Universiteti Str.

Doctor of Physical and Mathematical sciences

E-mail: diana.mtchedlishvili@gmail.com

${ }^{1}$ Associate Professor

${ }^{2}$ Professor

\section{Abstract}

In the paper is presented the fourth main boundary value problem of Dynamics of Thermoresiliency's Momentum theory. The problem states to find in the cylinder $D_{l}$ the regular solution of the system:

which satisfies the initial conditions:

$$
M\left(\partial_{x}\right) U-v \chi \theta-\chi^{0} \frac{\partial^{2} u}{\partial t^{2}}=\mathcal{H}, \quad \Delta \theta-\frac{1}{\vartheta} \frac{\partial \theta}{\partial t}-\eta \frac{\partial}{\partial t} \operatorname{div} u=\mathcal{H}_{7}
$$

$\forall x \in D: \lim _{t \rightarrow 0} U(x, t)=\varphi^{(0)}(x), \lim _{t \rightarrow 0} \theta(x, t)=\varphi_{7}^{(0)}(x), \lim _{t \rightarrow 0} \frac{\partial u(x, t)}{\partial t}=\varphi^{(1)}(x)$

and the boundary conditions:

$$
\forall(x, t) \in S_{l}: \lim _{D \ni x \rightarrow y \in S} P U=f, \quad \lim _{D \ni x \rightarrow y \in S}\{\theta\}_{S}^{ \pm}=f_{7} .
$$

The uniqueness theorem of the solution is proved for this problem.

Keywords: the main boundary value problem; initial conditions; boundary conditions; the uniqueness theorem of the solution.

\section{Introduction}

Let $D$ be a finite or infinite three-dimensional space with the compact boundary $S$ from the class $\Lambda_{2}(\alpha),(\alpha>0)$.

Denote by $D_{l}$ and $S_{l}$ cylinders $D_{l}=D \times l, S_{l}=S \times l$, respectively, where $l=[0, \infty)$. 
In the problems of Dynamics of Thermo-resiliency's Momentum theory any point of environment is characterized by seven quantities: a movement vector $-u=\left(u_{1}, u_{2}, u_{3}\right)$, a rotation vector $-\omega=\left(\omega_{1}, \omega_{2}, \omega_{3}\right)$ and a temperature deviation $-\theta$.

The main equations of the Thermo-resiliency's Momentum theory can be written in a matrix form as follows [1], [2]:

$$
M\left(\partial_{x}\right) U-v \chi \theta-\chi^{0} \frac{\partial^{2} u}{\partial t^{2}}=\mathcal{H}, \Delta \theta-\frac{1}{\vartheta} \frac{\partial \theta}{\partial t}-\eta \frac{\partial}{\partial t} \operatorname{div} u=\mathcal{H}_{7}
$$

where $M\left(\partial_{x}\right)$ is a matrix differential operator of the Momentum Resilience theory [3] and $\chi=$ $\left(\partial_{x_{1}}, \partial_{x_{2}}, \partial_{x_{3}}, 0,0,0\right), \chi^{0}=\left\|\chi_{i j}^{0}\right\|_{6 \times 6}, \chi_{i i}^{0}=\rho$ for $i=1,2,3, \chi_{i i}^{0}=\zeta$ for $i=4,5,6, \chi_{i j}^{0}=0$ for $i \neq j$, $\mathcal{H}=(-\rho F,-\rho \mathcal{Y}), \mathcal{H}_{7}=-\frac{1}{\vartheta} Q, \mathcal{U}=(u, \omega)$.

Let $\varphi^{(i)}=\left(\begin{array}{l}1 \\ \varphi\end{array}(i), \underset{\varphi}{2}(i)\right)$ for $i=0,1$, where ${ }_{\varphi}^{k}(i)=\left(\varphi_{1}^{k_{(i)}}, \varphi_{2}^{k_{(i)}}, \varphi_{3}^{k_{(i)}}\right)$ for $k=1,2$ and $\varphi_{7}^{(i)}$ for $i=0,1$ be functions given in the area $\bar{D}$, while $f=\left(f^{(1)}, f^{(2)}\right), f^{(i)}=\left(f_{1}^{(i)}, f_{2}^{(i)}, f_{3}^{(i)}\right)$ for $i=1,2$ and $f_{7}$ are functions given on $S_{l}$.

\section{Definition}

Vector-function $U=(u, \omega, \theta)$ is called as regular in the area $D_{l}^{+}$if $U \in C^{1}\left(\bar{D}_{l}^{+}\right) \cap C^{2}\left(D_{l}^{+}\right)$for $\forall t \in l$ and $B(\partial x, \partial t) \mathrm{U}$ is integrable in the area $D^{+}$.

Analogously, vector-function $U=(u, \omega, \theta)$ is called as regular in the area $D_{l}^{-}$if $U \in C^{1}\left(\bar{D}_{l}^{-}\right) \cap$ $C^{2}\left(D_{l}^{-}\right)$for $\forall t \in l$ and $B(\partial x, \partial t) \mathrm{U}$ is integrable in the area $D^{-} \cap \mathcal{M}(\mathrm{o}, \delta)$ for any number $\delta>0$ and

$$
|U(x, \tau)| \leq \frac{c(t)}{1+|x|^{2}},\left|\frac{\partial U(x, \tau)}{\partial t}\right| \leq \frac{c(t)}{1+|x|^{2}},\left|\frac{\partial U(x, \tau)}{\partial x_{i}}\right| \leq \frac{c(t)}{1+|x|^{2}},
$$

where $B(\partial x, \partial t)$ is an operator standing on the left side of the system (1) and is written in the form of a matrix differential operator.

In the paper is studied the following problem of Dynamics of Thermo-resiliency's Momentum theory: to find in the cylinder $D_{l}$ the regular solution of the system (1) which satisfies the initial conditions:

$\forall x \in D: \lim _{t \rightarrow 0} U(x, t)=\varphi^{(0)}(x), \lim _{t \rightarrow 0} \theta(x, t)=\varphi_{7}^{(0)}(x), \lim _{t \rightarrow 0} \frac{\partial u(x, t)}{\partial t}=\varphi^{(1)}(x)$

and the boundary conditions:

$$
\forall(x, t) \in S_{l}: \lim _{D \ni x \rightarrow y \in S} P U=f, \quad \lim _{D \ni x \rightarrow y \in S}\{\theta\}_{S}^{ \pm}=f_{7} .
$$

Here, $P=P(\partial x, n)$ is an operator of thermo-momentary voltage:

$$
P(\partial x, n) U=T(\partial x, n) U-v e \theta,
$$

where $T(\partial x, n)$ is an operator of momentary voltage [3], $e=\left(n_{1}, n_{2}, n_{3}, 0,0,0\right), \mathcal{U}=(u, \omega)$ and $\mathrm{n}\left(n_{1}, n_{2}, n_{3}\right)$ is a normal of the surface $S$.

\section{The main result}

The following uniqueness theorem is true:

Theorem. In the cylinder $D_{l}^{ \pm}$the regular solution of the homogenous problem, corresponding to the above stated problem, is identical to 0 .

The proof of the theorem. Let $U=(\mathcal{U}, \theta)$ be a regular solution in $D_{l}^{+}$of the homogenous equation corresponding to (1). Then, the following formula is true:

$$
\begin{aligned}
\frac{\partial}{\partial t} \int_{D^{+}}\left\{\frac{1}{2} \sum_{i=1}^{6} \chi_{i i}^{0}\left|\frac{\partial U_{i}}{\partial t}\right|^{2}+\frac{1}{2} E(\mathcal{U}, \mathcal{U})+\frac{v}{2 \vartheta \eta}|\theta|^{2}\right\} d x+ \\
+\frac{v}{\eta} \int_{D^{+}}|\operatorname{grad} \theta|^{2} d x=\int_{S}\left\{\frac{\partial u}{\partial t} P U-\frac{v}{\eta} \theta \frac{\partial \theta}{\partial n}\right\} d S,
\end{aligned}
$$

where $E(\mathcal{U}, \mathcal{U})$ is a positively defined form [3].

For the regular solution of the homogenous problem in $D_{l}^{+}$the right side of (3) is equal to 0 . Hence, the left side of it is also equal to 0 , from which follows that $U=0, \theta=0$. So, $U=0$.

Now, let $U=(\mathcal{U}, \theta)$ be a regular solution of the homogenous problem in $D_{l}^{-}$, corresponding to the system (1). We can write (3) for $D^{-} \cap \mathcal{M}(0, z)$ as follows: 
where $z$ is a sufficiently large number.

$$
\begin{gathered}
\frac{\partial}{\partial t} \int_{D^{-} \cap \mathcal{M}(0, z)}\left\{\frac{1}{2} \sum_{i=1}^{6} \chi_{i i}^{0}\left|\frac{\partial u_{i}}{\partial t}\right|^{2}+\frac{1}{2} E(\mathcal{U}, \mathcal{U})+\frac{v}{2 \vartheta \eta}|\theta|^{2}\right\} d x+ \\
+\frac{v}{\eta} \int_{D^{-} \cap \mathcal{M}(0, z)}|\operatorname{grad} \theta|^{2} d x=\int_{C(0, z)}\left\{\frac{\partial \mathcal{U}}{\partial t} P U+\frac{v}{\eta} \theta \frac{\partial \theta}{\partial n}\right\} d S,
\end{gathered}
$$
that

Considering the conditions (2) and taking the limit of the above equation as $z \rightarrow \infty$, we get

$$
\frac{\partial}{\partial t} \int_{D^{-}}\left\{\frac{1}{2} \sum_{i=1}^{6} \chi_{i i}^{0}\left|\frac{\partial \mathcal{U}_{i}}{\partial t}\right|^{2}+\frac{1}{2} E(\mathcal{U}, \mathcal{u})+\frac{v}{2 \vartheta \eta}|\theta|^{2}\right\} d x+\frac{v}{\eta} \int_{D^{-}}|\operatorname{grad} \theta|^{2} d x=0,
$$

from which, using the homogeneity of the initial conditions, we have:

Thus, the theorem is proved.

$$
U=0 \text {. }
$$

\section{Conclusion}

The main task was to prove the uniqueness theorem of the solution of the fourth main boundary value problem of Dynamics of Thermo-resiliency's Momentum theory. In the cylinder $D_{l}$ was found the regular solution of the system:

$$
M\left(\partial_{x}\right) U-v \chi \theta-\chi^{0} \frac{\partial^{2} u}{\partial t^{2}}=\mathcal{H}, \Delta \theta-\frac{1}{\vartheta} \frac{\partial \theta}{\partial t}-\eta \frac{\partial}{\partial t} \operatorname{div} u=\mathcal{H}_{7},
$$

which satisfies the following initial and boundary conditions:

$$
\begin{gathered}
\forall x \in D: \lim _{t \rightarrow 0} U(x, t)=\varphi^{(0)}(x), \lim _{t \rightarrow 0} \theta(x, t)=\varphi_{7}^{(0)}(x), \lim _{t \rightarrow 0} \frac{\partial u(x, t)}{\partial t}=\varphi^{(1)}(x) ; \\
\forall(x, t) \in S_{l}: \lim _{D \ni x \rightarrow y \in S} P U=f, \lim _{D \ni x \rightarrow y \in S}\{\theta\}_{S}^{ \pm}=f_{7} .
\end{gathered}
$$

\section{References:}

1. Novatski, V. (1975). Resilience theory. Moscow (in Russian)

2. Aghniashvili, M. (1976). Some boundary value problems of thermo-momentum resilience theory. Tbilisi (in Russian)

3. Kupradze, V., Gegelia, T., Basheleishvili, M., Burtchuladze, T. (1976). Three-dimensional problems of mathematical resilience theory and thermo-resiliency. Tbilisi (in Russian) 